\title{
Non-steroidal anti-inflammatory drugs do not influence the urinary testosterone/epitestosterone glucuronide ratio
}

\author{
Jonas Lundmark, Nina Gårevik, John-OlofThörngren, Mats Garle, Lena Ekström, Anders Rane and \\ Jenny J. Schulze*
}

Division of Clinical Pharmacology, Department of Laboratory Medicine, Karolinska Institutet, Stockholm, Sweden

Edited by:

Russell J. Borski, North Carolina State University, USA

Reviewed by:

Moshe Finel, University of Helsinki,

Finland

Rosa Ventura, Fundació Institut

Hospital del Mar d'Investigacions

Mèdiques, Spain

*Correspondence:

Jenny J. Schulze, Clinical

Pharmacology C1:68, Karolinska

University Hospital, Huddinge, 14186

Stockholm, Sweden.

e-mail: jenny.schulze@ki.se
The UDP Glucuronosyl Transferase (UGT) enzymes are important in the pharmacokinetics, and conjugation, of a variety of drugs including non-steroidal anti-inflammatory drugs (NSAIDs) as well as anabolic androgenic steroids (AAS). Testosterone glucuronidation capacity is strongly associated with a deletion polymorphism in the UGT2B17 gene. As the use of high doses of NSAIDs has been observed in athletes there is a risk for a drugdrug interaction that may influence the doping tests for AAS. In vitro studies show inhibitory potential on UGT2B7, 2B15, and 2B17 enzymes by NSAIDs. The aim of this study was to investigate if concomitant use of NSAIDs and a single dose of testosterone enanthate would affect the excretion rate of testosterone and epitestosterone glucuronide (TG and $E G)$ as well as the T/E ratio, thereby affecting the outcome of the testosterone doping test. The study was designed as an open, randomized, cross-over study with subjects being their own control. The 23 male healthy volunteers, with either two, one or no allele (ins/ins, ins/del, or del/del) of the UGT2B17 gene, received the maximum recommended dose of NSAID (Ibuprofen or Diclofenac) for 6 days. On day three, $500 \mathrm{mg}$ of testosterone enanthate was administered. Spot urine samples were collected for 17 days. After a wash-out period of 4 months the volunteers received $500 \mathrm{mg}$ testosterone enanthate only, with subsequent spot urine collection for 14 days. The glucuronides of testosterone and epitestosterone were quantified. NSAIDs did not affect the excretion of TG or EG before the administration of testosterone. The concomitant use of NSAIDs and testosterone slightly increased the TG excretion while the EG excretion was less suppressed compared to testosterone use only. The effects of the NSAIDs on the TG and EG excretion did not differ between the UGT2B17 genotype groups. In conclusion, the outcome of testosterone doping tests does not seem to be affected by the use of NSAIDs.

Keywords: NSAID, testosterone, epitestosterone, diclofenac, ibuprofen, UGT2B17, T/E ratio

\section{INTRODUCTION}

Testosterone is one of the most commonly abused anabolic androgenic steroids (AAS) within doping in sports and for enhancement of physical performance (Handelsman and Heather, 2008). Testosterone and other anabolic compounds are prohibited in sports by the World Anti-Doping Agency (WADA). In 2011 anabolic agents represented the most frequently reported adverse analytical findings and atypical findings (59.4\%) by accredited doping laboratories. Among these, elevated testosterone/epitestosterone ratios represented $60.0 \%$ of the findings, although only $10 \%$ of these were adverse analytical findings (WADA, 2011).

To discriminate exogenous testosterone from testosterone of endogenous origin the urinary ratio of testosterone glucuronide (TG) to epitestosterone glucuronide (EG) (T/E ratio) is used (Donike et al., 1983, 1985). Based on population studies (Donike et al., 1983, 1985) a normal T/E ratio would be $\sim 1.0$ and a T/E ratio above six was initially considered suspicious of doping. However, additional knowledge showed that Asian individuals excreted low amounts of TG, and as a result low $\mathrm{T} / \mathrm{E}$ ratios increasing the risk of false negative doping test results (Park et al., 1990; de la Torre et al., 1997). Due to these findings the T/E ratio was lowered to 4.0 in 2004 .

Testosterone is inactivated, and excreted in urine, mainly as glucuronide conjugates, the formation of which is catalyzed by UDP-glucuronosyltransferases (UGTs). These enzymes play a key role in the homeostasis of a number of endogenous molecules including steroid hormones (Belanger et al., 2003). The UGT super family is subdivided into UGT1A, UGT2A, and UGT2B families based on sequence identity (Mackenzie et al., 1997). In humans UGTs $2 \mathrm{~B} 7,2 \mathrm{~B} 15$, and $2 \mathrm{~B} 17$ are the main catalysts of the glucuronidation of androgens and their metabolites (Belanger et al., 2003). Testosterone glucuronidation is mainly dependent on UGT2B17 and to a lesser extent UGT2B15 (Turgeon et al., 2001) whereas epitestosterone is conjugated by UGT2B7 (Coffman et al., 1998; Sten et al., 2009a).

The human UGT2B genes are clustered on chromosome 4q1321.1 and encode seven functional enzymes: UGT2B4, B7, B10, B11, B15, B17, and B28 (Guillemette, 2003). In vivo, UGT2B17 has been identified as the main enzyme in testosterone glucuronidation where a gene deletion in UGT2B17 was associated by us with 
low, or negligible, excretion of testosterone in urine (Jakobsson et al., 2006). All subjects devoid of UGT2B17 had a T/E ratio below 0.4 . This polymorphism was considerably more common in a Korean Asian than in a Swedish Caucasian population, with 66.7 and 9.3\% deletion/deletion (del/del) homozygotes, respectively (Jakobsson et al., 2006). This correlates to earlier findings of low T/E in Asians as described above. Further, after testosterone administration individuals devoid of the UGT2B17 gene rarely exceed this cut-off ratio (Schulze et al., 2008b). Individuals carrying one (ins/del) or two (ins/ins) copies of this gene clearly passed the cut-off ratio and some even exceeded the cut-off level prior to challenge with testosterone.

The UGT enzymes are important in the pharmacokinetics, and conjugation, of a variety of drugs including non-steroidal antiinflammatory drugs (NSAIDs) (Davies, 1998; Kuehl et al., 2005). NSAIDs are a class of therapeutic agents used in the treatment of pain and inflammation and are commonly used by athletes. In fact, according to recent studies, inappropriate use of high doses and concomitant use of several different NSAIDs has been observed in athletes (Alaranta et al., 2008; Warden, 2009). Since steroids and NSAIDs are both inactivated by UGT enzymes there is a risk for a drug-drug interaction (Kiang et al., 2005). In vitro studies show inhibitory potential on UGT2B7 (Mano et al., 2007), 2B15 and 2B17 enzymes by NSAIDs (Sten et al., 2009b). In the latter study both diclofenac and ibuprofen inhibited testosterone glucuronidation in liver microsomes, as well as recombinant UGT2B15 and UGT2B17 enzymes. However, epitestosterone glucuronidation activity in human liver microsomes was largely insensitive to ibuprofen and diclofenac. To our knowledge this has not been studied in vivo.

The aim of this study was to investigate in healthy male volunteers whether a concomitant use of NSAIDs (Ibuprofen or Diclofenac) and a single dose of testosterone enanthate would affect the excretion rate of TG and EG, and hence the T/E ratio, to the extent that it could affect the outcome of the testosterone doping test.

\section{MATERIALS AND METHODS \\ SUBJECTS}

The target group was healthy male volunteers aged $18-50$ years. A total number of 33 subjects were genotyped for the UGT2B17 deletion polymorphism to fill the pre-determined number of approximately 10 subjects in each of the three different genotype panels (ins/ins, ins/del and del/del). All subjects originated from different ethnicities and therefore the genotype frequencies in this sample are not representative of any particular population. In this particular genotyped population group $28 \%$ were homozygous for the gene deletion (del/del), 33\% were heterozygous (ins/del), and 39\% had two copies of the gene (ins/ins). In total, eight del/del, seven ins/del, and eight ins/ins participants completed the study. Study population characteristics are presented in Table 1. In addition to genotyping, all subjects underwent a medical examination including laboratory tests before enrollment to exclude the possibility of any disease. No pharmaceutical compounds other than those in this study were allowed. Further inclusion-criteria included a negative screening for illegal drugs, AAS, HIV, and hepatitis $\mathrm{B}$ or $\mathrm{C}$ virus. For inclusion it was also
Table 1 | Study population characteristics at screening.

\begin{tabular}{lllll}
\hline $\begin{array}{l}\text { UGT2B17 } \\
\text { Genotype }\end{array}$ & Age (years) & Height $(\mathbf{c m})$ & Weight $(\mathbf{k g})$ & BMI $\left(\mathbf{k g} / \mathbf{m}^{\mathbf{2}}\right)$ \\
\hline del/del $(n=8)$ & $29.2 \pm 4.3$ & $180 \pm 6.9$ & $80.6 \pm 9.7$ & $24.8 \pm 2.7$ \\
ins/del $(n=7)$ & $31.1 \pm 4.7$ & $179 \pm 6.1$ & $81.4 \pm 9.2$ & $25.4 \pm 2.8$ \\
ins/ins $(n=8)$ & $28.1 \pm 5.1$ & $181 \pm 5.2$ & $79.6 \pm 6.1$ & $24.3 \pm 2.3$ \\
\hline
\end{tabular}

required that the subject was not a member of any organization belonging to the Swedish Sports Confederation, had been diagnosed and/or treated for a malignancy within the past 5 years or having a known allergy to the study substances. All participants gave informed consent consistent with the approval of the Ethics Review Board at Karolinska Institutet in Stockholm. Two subjects were excluded prior to the start of the study due to concomitant medication. The genotyped subjects that did not participate were dropouts $(n=8)$. The study (protocol number 2007-002655-16) was conducted according to the Helsinki declaration and the $\mathrm{ICH}$ Harmonized Tripartite Guideline for Good Clinical Practice.

\section{GENOTYPING OF UGT2B17}

The copy number of UGT2B17 was determined in the 23 males included in the study. The numbers of UGT2B17 genes quantified with a real-time PCR analysis, using the expression of albumin as an endogenous control. The genotyping methods are based on the $5^{\prime}$-nuclease activity method ( $\operatorname{TaqMan}^{\circledR}$ ) employing two primers and two fluorescent labeled probes in a real-time based assay as earlier described (Schulze et al., 2008a).

\section{DESIGN}

The study was designed as an open, randomized, cross-over study with subjects being their own control (Figure 1). In the Phase I, starting 3 days before $(-3)$ administration of Testoviron ${ }^{\circledR}$-Depot, subjects received either diclofenac (Voltaren $\left.{ }^{\circledR}, n=13\right) 50 \mathrm{mg} \times 3$ or ibuprofen $\left(\right.$ Ipren $\left.^{\circledR}, n=10\right) 400 \mathrm{mg} \times 3$ for six consecutive days (ending on day-2).

On day-0 all participants from the different genotype panels were given a single dose of $500 \mathrm{mg}$ testosterone enanthate in ricinus oil as a single intramuscular dose of Testoviron ${ }^{\circledR}$ - Depot (kindly provided by Bayer Schering Pharma) equivalent to $360 \mathrm{mg}$ testosterone. Before administration of the testosterone, urine samples were collected during 4 days (days-3, $-2,-1$, and 0 ). Samples of urine were further collected for 14 days, all between 06-10 am. In the Phase II all participants were given a single dose of $500 \mathrm{mg}$ testosterone enanthate in ricinus oil as a single intramuscular dose of Testoviron ${ }^{\circledR}$-Depot equivalent to $360 \mathrm{mg}$ testosterone. A baseline urine sample was collected before the injection and further for 14 days after the injection, all between 06:00 and 10:00 a.m. The wash-out period between the two phases was at least 4 months. Adverse drug reactions (ADRs) were monitored from inclusion of each phase until day-14 after administration of testosterone. No major ADRs were registered. No follow-up was needed.

\section{BLOOD AND URINE SAMPLES}

Venous blood was obtained from the cubital vein and collected in EDTA tubes for DNA extraction. The urine samples were collected 


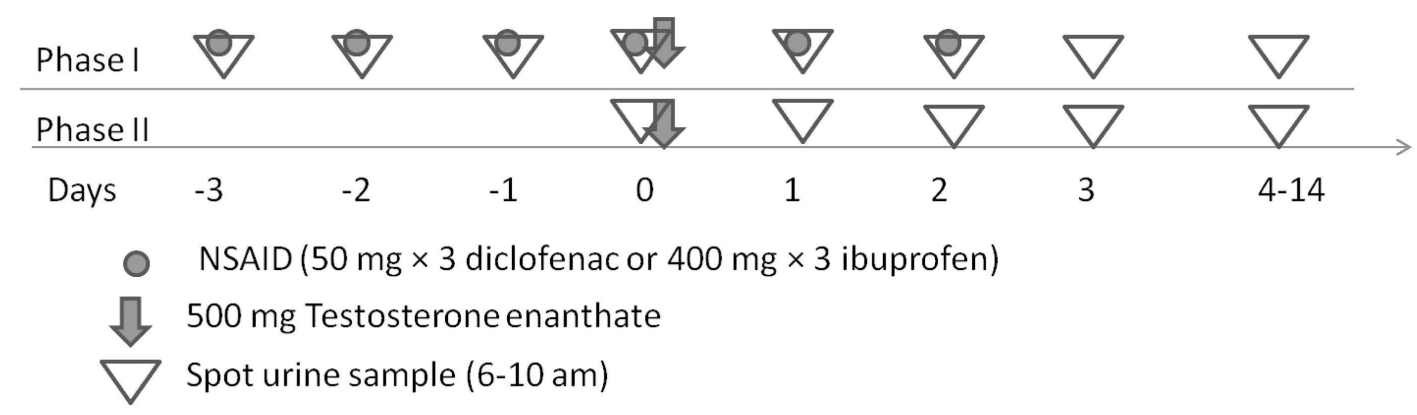

FIGURE 1 | Illustration of the open, randomized, cross-over study with subjects being their own control. The wash-out period was at least 4 months.

Table 2 | Baseline urinary androgen glucuronide levels (A) before and after 3 days of NSAID administration Phase I and (B) in Phase II.

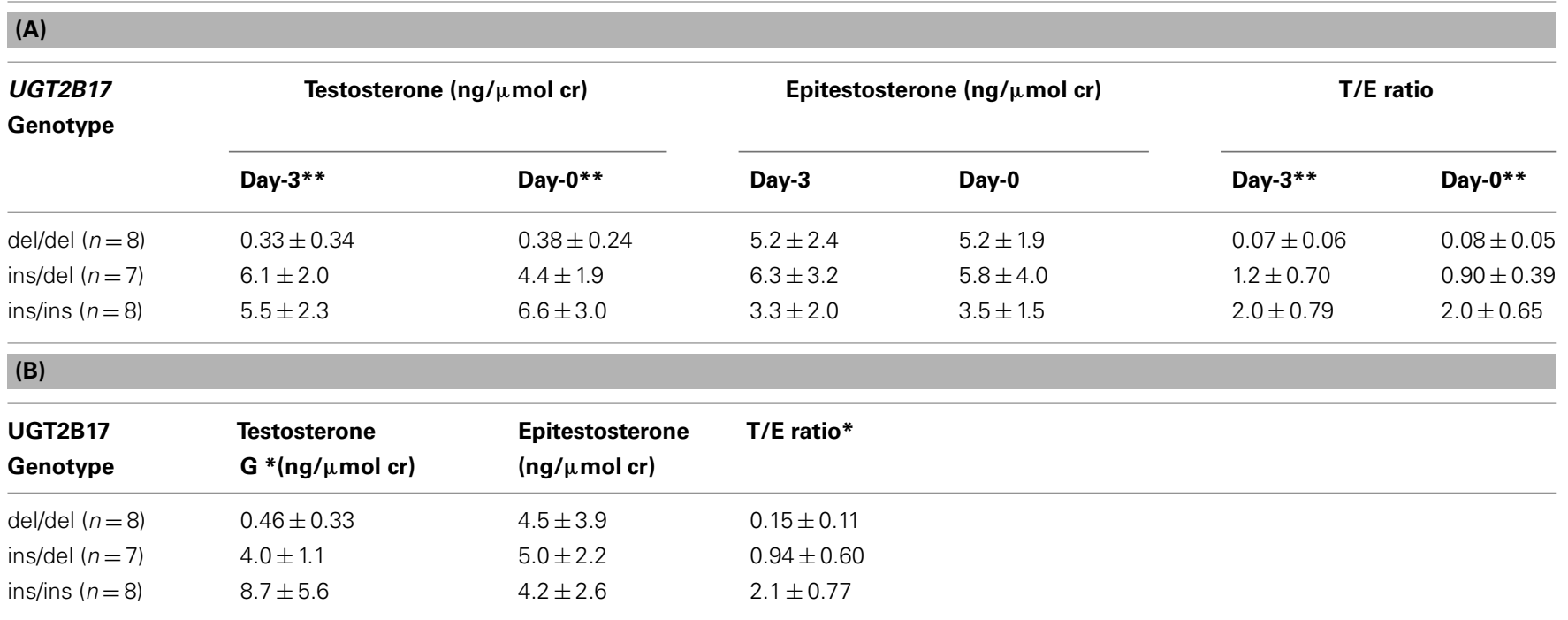

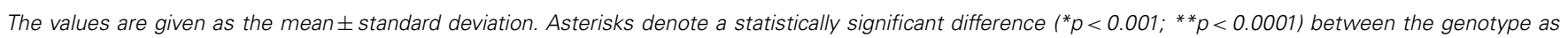
determined by ANOVA.

and kept refrigerated for maximum $48 \mathrm{~h}$ and then frozen at $-20^{\circ} \mathrm{C}$. All samples were collected between 06:00 and 10:00 a.m.

\section{URINE ANALYSES}

Urinary unconjugated steroids and steroid glucuronides were determined using liquid chromatography-mass spectrometry (LC/MS) as described previously (Schulze et al., 2011). The method was modified to only quantify the glucuronides of testosterone and epitestosterone. The day-to-day variation of the instrument was minimized using the mixture of authentic standards analyzed with every batch of samples.

\section{DATA ANALYSES}

Integration, calibration, and data evaluation was performed by the TargetLynx software (Waters Associates, Manchester, UK).

The between-subject variation in urine dilution was corrected for by dividing the concentration values by the urinary creatinine (cr) concentration, which was determined by colorimetric analysis (DRI Creatinine-Detect Test; Thermo Fisher Scientific, Waltham, MA, USA). Statistical analyses for comparison of hormonal levels of TG, EG, and T/E were performed with Student's two-tailed $t$ test or Mann-Whitney $U$ test according to distribution of the data. When comparing three groups; ANOVA with Tukey's post hoc test or Kruskal-Wallis with Dunn's post hoc test according to distribution. $p<0.05$ regarded as significant.

\section{RESULTS}

\section{BASELINE URINARY STEROIDS}

The average baseline urinary glucuronidated testosterone (TG) and epitestosterone (EG) concentrations and T/E ratios for Phase I before and after 3 days of NSAID administration are presented in Table 2. Neither the TG levels, EG levels nor the T/E ratio were affected by NSAID administration. For the ins/del group the TG levels decreased slightly after 3 days of NSAID administration, however, this decrease was not statistically significant $(p=0.13)$. As expected, the TG levels and $\mathrm{T} / \mathrm{E}$ ratio differed significantly between the del/del group and the other two genotype groups. There was no difference in the EG levels. No difference in baseline values between individuals aimed for receiving ibuprofen or diclofenac was observed. 

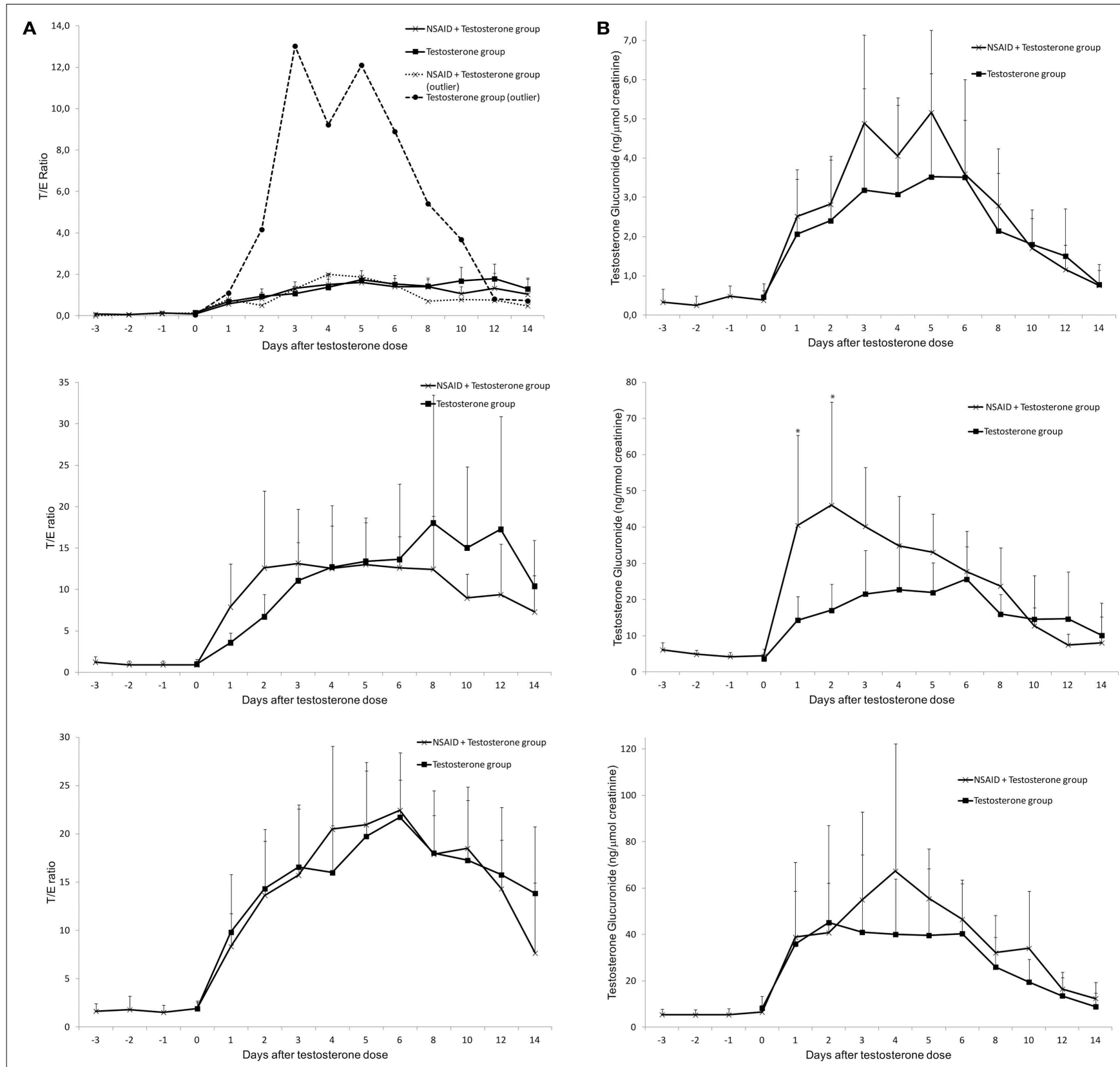

FIGURE 2 | Average urinary testosterone/epitestosterone ratios (A) and urinary testosterone glucuronide excretion ( $\mathrm{ng} / \mu \mathrm{mol}$ creatinine) (B) during 14 days in UGT2B17 del/del (top), ins/del (middle) and ins/ins (bottom) genotype groups of healthy volunteer males. The subjects were followed during two administration cycles with at least 4 months wash-out

The average baseline T/E ratios for Phase II are presented in Table 2. Similar to Phase I the TG levels and the T/E ratio differed significantly between the genotype groups.

\section{URINARY STEROID PROFILE AFTER TESTOSTERONE ADMINISTRATION}

The T/E ratios in Phase I (NSAID + T) and Phase II (T only) for the different genotype panels after testosterone dose are shown in Figure 2A. There were no statistically significant differences

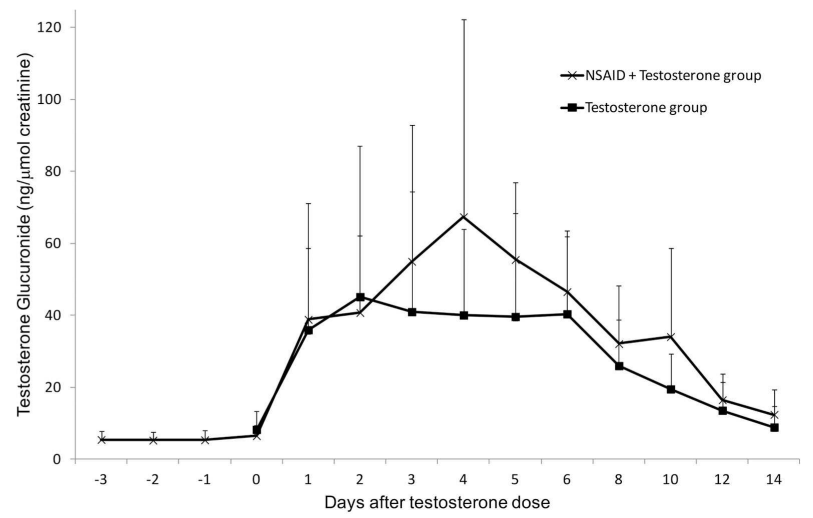

between the cycles. During one cycle NSAID was administered for 6 days (day-3 to day-2). In both cycles an intramuscular dose of $500 \mathrm{mg}$ testosterone enanthate was administered on day-0. Asterisks denote statistically significant differences between the groups $\left({ }^{*} p<0.05 ;{ }^{*} p<0.01\right)$. Vertical bars denote standard deviations.

between Phase I and Phase II. In the del/del group there was one individual with EG levels decreasing much less in Phase I compared to Phase II, while his TG levels were similar in both Phase I and Phase II. This individual is shown as a dotted line in Figure 2A (top).

The TG increased slightly more in Phase I (NSAID + T) compared to Phase II ( $\mathrm{T}$ only) after dose in all genotype groups (Figure 2B). However the increase was only significant in the 
ins/del genotype group on day-1 $(p=0.02)$ and day-2 $(p=0.05)$. Taken all genotype groups together the maximum increase in TG excretion compared to baseline was about $80 \%$ higher in Phase I $(\mathrm{NSAID}+\mathrm{T})$ compared to Phase II (T only). There was no statistically significant difference between the diclofenac group compared to the ibuprofen group.

The excretion of EG decreased steadily to low levels after the testosterone dose as expected. The decrease was, however, more pronounced in Phase II ( $\mathrm{T}$ only) compared to Phase I $(\mathrm{NSAID}+\mathrm{T})$ in all genotype groups the first 5 days after the testosterone dose. This corresponds to the time the study subjects still had relatively high concentrations of NSAID in their circulation. As the EG excretion is not dependent on UGT2B17 genotype the three UGT2B17 genotype panels were grouped for increased statistical power (Figure 3). On days 1-5 after the testosterone dose, the EG concentrations were significantly less suppressed in Phase I (+NSAID) compared to Phase II $(p=0.04-0.003)$ with the exception of day-2 $(p=0.08)$. No difference was seen between the diclofenac and the ibuprofen group.

Taken together the results indicate that NSAID slightly induces TG and decreases suppression of EG excretion, hence there will be no effect on the $\mathrm{T} / \mathrm{E}$ ratio.

\section{DISCUSSION}

In this study, for the first time, we aimed to study if NSAIDs affect testosterone glucuronidation, epitestosterone glucuronidation and hence T/E ratios in vivo. Based on previous in vitro data (Sten et al., 2009b) the hypothesis was that NSAIDs would interact with the glucuronidation of testosterone but not epitestosterone leading to lower TG excretion and lower T/E ratios. However, the opposite was seen, with a minor increase in TG and less suppression of EG excretion leading to unchanged T/E ratios.

In our in vitro study (Sten et al., 2009b) the $\mathrm{IC}_{50}$ values for the inhibition of testosterone glucuronidation by ibuprofen was 213 (95\% CI; 129-354) $\mu \mathrm{M}$ and by diclofenac 64 (95\% CI;5380 ) in human liver microsomes (Sten et al., 2009b). Therapeutic plasma concentrations for ibuprofen are $73-146 \mu \mathrm{M}$, which are in the same range as the IC50 value. For diclofenac the therapeutic plasma concentrations are considerably lower (about $5 \mu \mathrm{M}$ ) than the in vitro IC50 value. We still decided to include diclofenac in the in vivo study, as this is a very common NSAID used by athletes.

It is not straight forward to predict inhibitory interactions involving glucuronidated drugs from in vitro data (Miners et al., 2006) and references therein. We have shown that testosterone glucuronidation rate in vitro in liver microsomes was only twofold higher in livers with one or two copies of the UGT2B17 gene compared to UGT2B17 del/del livers (Jakobsson et al., 2006). In vivo, urinary levels of TG in UGT2B17 ins/ins and ins/del individuals are over 10 times higher than TG levels in UGT2B17 del/del individuals (this study; Jakobsson et al., 2006; Schulze et al., 2008b). In addition, in our in vitro experiment only liver microsomes and recombinant enzymes were studied (Sten et al., 2009b), while the UGT2B enzymes are highly expressed in several different tissues (Nakamura et al., 2008).

Our intention with this study was aimed at mimicking a situation that doping laboratories may face when athletes are taking NSAIDs. We conclude that NSAIDs, even in large doses, will not have any significant effect on testosterone doping tests results,

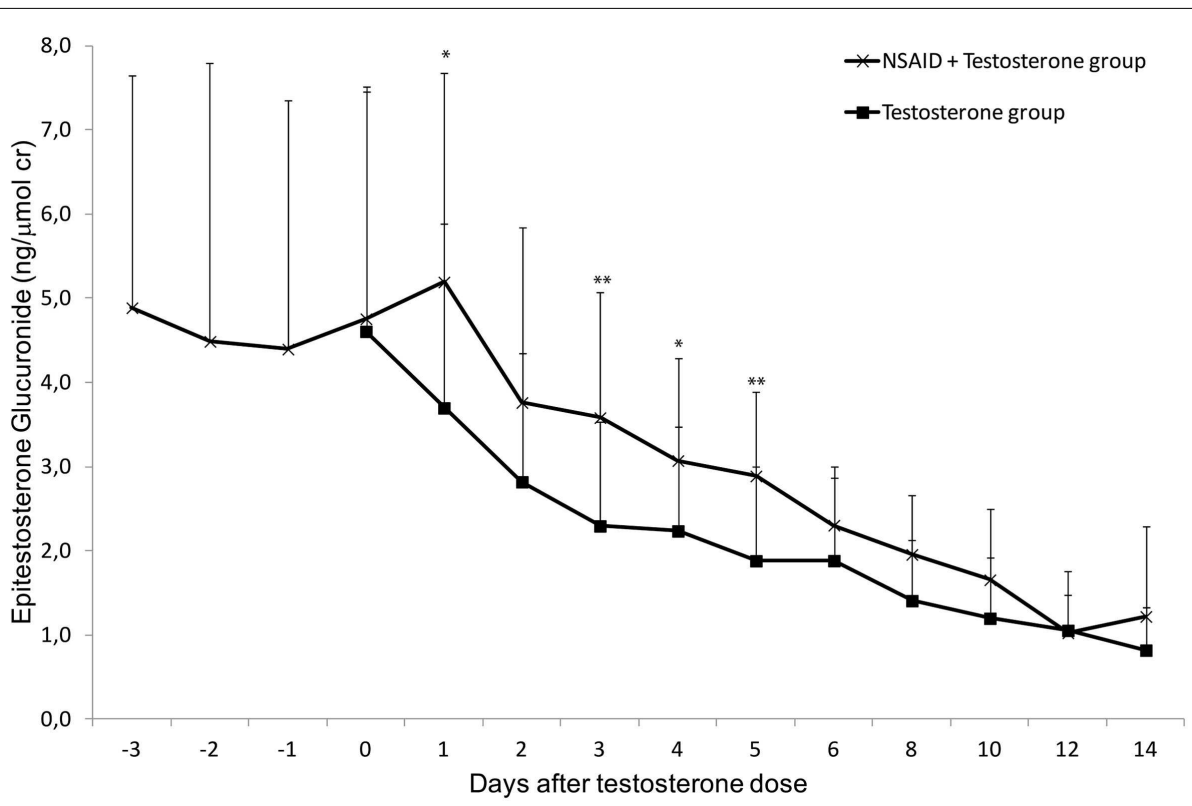

FIGURE 3 | Average urinary epitestosterone glucuronide excretion ( $\mathrm{ng} / \mu \mathrm{mol}$ creatinine) during $\mathbf{1 4}$ days in healthy volunteer males. The subjects were followed during two administration cycles with at least 4 months wash-out between the cycles. During one cycle NSAID was administered for 6 days (day-3 to day-2). In both cycles an intramuscular dose of $500 \mathrm{mg}$ testosterone enanthate was administered on day- 0 . Asterisks denote statistically significant differences between the groups $\left({ }^{*} p<0.05\right.$ : $\left.{ }^{* *} p<0.01\right)$ Vertical bars denote standard deviations. 
at least not with injected testosterone esters. Oral formulations of testosterone are metabolized in the liver to a much higher extent than injectable testosterone esters. It would be interesting to investigate if oral formulations of testosterone together with NSAID would show different results. It is possible that this setting would be more similar to our in vitro investigation where liver microsomes were used (Sten et al., 2009b).

To our knowledge the interaction of NSAID with the glucuronidation enzymes has not been extensively studied in vivo. Van der Logt et al. (2004) demonstrated increased UGT enzyme activities in rats treated with ibuprofen in intestine but not in liver.

The plasma concentrations of ibuprofen and diclofenac are known to be highly dependent on polymorphisms in CYP2C9 and CYP2C8 (Garcia-Martin et al., 2004; Zhou et al., 2010). Indeed, the TG excretion increased more than $20 \%$ in 16 study subjects, was unchanged in six and was decreased in one subject after the testosterone injection with concomitant use of NSAID compared to a testosterone injection only.

We chose to use a large dose of testosterone enanthate in order to have plasma concentrations as close as possible to our previous

\section{REFERENCES}

Alaranta, A., Alaranta, H., and Helenius, I. (2008). Use of prescription drugs in athletes. Sports Med. 38, 449-463.

Belanger, A., Pelletier, G., Labrie, F., Barbier, O., and Chouinard, S. (2003). Inactivation of androgens by UDPglucuronosyltransferase enzymes in humans. Trends Endocrinol. Metab. 14, 473-479.

Coffman, B. L., King, C. D., Rios, G. R., and Tephly, T. R. (1998). The glucuronidation of opioids, other xenobiotics, and androgens by human UGT2B7Y(268) and UGT2B7H(268). Drug Metab. Dispos. 26, 73-77.

Davies, N. M. (1998). Clinical pharmacokinetics of ibuprofen. The first 30 years. Clin. Pharmacokinet. 34, 101-154.

de la Torre, X., Segura, J., Yang, Z., Li, Y., and Wu, M. (1997). "Testosterone detection in different ethnic groups," in Recent Advances in Doping Analysis (4), eds W. Schänzer, H. Geyer, A. Gotzman, and U. Mareck-Engelke (Köln: Sport und Buch Strauss), 71-89.

Donike, M., Adamietz, B., Opfermann, G., Schänzer, W., Zimmermann, J., and Mandel, F. (1985). "Die normbereiche für testosteron- und epitestosterone urinspiegel sowie des testosteron-/epitestosteronquotienten," in Training und Sport zur Prävention und Rehabilitation in der Technisierten Umwelt, eds $\mathrm{H}$. Mellerowicz, I. W. Franz, and W.
Noack (Berlin: Springer Verlag), 503-507.

Donike, M., Bärwald, K., Klostermann, K., Schänzer, W., and Zimmermann, J. (1983). "Detection of exogenous testosterone," in Sport:Leistung und Gesundheit, Kongressbd. Dtsch Sportarztekongress, eds H. Heck, W. Hollmann, H. Liesen, and R. Rost (Köln: Deutcher Ärtzte-Verlag), 293-298.

Garcia-Martin, E., Martinez, C., Tabares, B., Frias, J., and Agundez, J. A. (2004). Interindividual variability in ibuprofen pharmacokinetics is related to interaction of cytochrome P450 2C8 and 2C9 amino acid polymorphisms. Clin. Pharmacol. Ther. 76, 119-127.

Guillemette, C. (2003). Pharmacogenomics of human UDP-glucuronosyltransferase

enzymes. Pharmacogenomics J. 3, 136-158.

Handelsman, D. J., and Heather, A. (2008). Androgen abuse in sports. Asian J. Androl. 10, 403-415.

Jakobsson, J., Ekstrom, L., Inotsume, N., Garle, M., Lorentzon, M., Ohlsson, C., et al. (2006). Large differences in testosterone excretion in Korean and Swedish men are strongly associated with a UDP-glucuronosyl transferase $2 \mathrm{~B} 17$ polymorphism. J. Clin. Endocrinol. Metab. 91, 687-693.

Jenkinson, C., Petroczi, A., Barker, J., and Naughton, D. P. (2012a). Dietary green and white teas suppress UDPglucuronosyltransferase UGT2B17

in vitro investigation (Sten et al., 2009b), and also, since our study group was quite small, to be able to detect any changes in TG and EG excretion. It would be interesting to use different formulations of testosterone rather than different types of NSAIDs.

Several in vitro studies have shown UGT2B inhibition with different types of food or drink (Jenkinson et al., 2012a,b) or drugs (Sten et al., 2009b) and speculated that this will affect the testosterone excretion and testosterone doping tests. Our in vivo study does not confirm previous in vitro results, rather the opposite, and this should be taken into consideration when extrapolating in vitro results to the in vivo situation.

In conclusion, the concomitant use of NSAIDs and testosterone esters slightly increases the TG excretion while the EG excretion is less suppressed compared to testosterone use only. The T/E ratios are not affected by NSAID intake either before or after the testosterone administration.

\section{ACKNOWLEDGMENTS}

This study was supported by the World Anti-Doping Agency (WADA and the Swedish National Centre for Research in Sports.

mediated testosterone glucuronidation. Steroids 77, 691-695.

Jenkinson, C., Petroczi, A., and Naughton, D. P. (2012b). Red wine and component flavonoids inhibit UGT2B17 in vitro. Nutr. J. 11, 67.

Kiang, T. K., Ensom, M. H., and Chang, T. K. (2005). UDPglucuronosyltransferases and clinical drug-drug interactions. Pharmacol. Ther. 106, 97-132.

Kuehl, G. E., Lampe, J. W., Potter, J. D., and Bigler, J. (2005). Glucuronidation of nonsteroidal antiinflammatory drugs: identifying the enzymes responsible in human liver microsomes. Drug Metab. Dispos. 33, 1027-1035.

Mackenzie, P. I., Owens, I. S., Burchell, B., Bock, K. W., Bairoch, A., Belanger, A., et al. (1997). The UDP glycosyltransferase gene superfamily: recommended nomenclature update based on evolutionary divergence. Pharmacogenetics 7 , 255-269.

Mano, Y., Usui, T., and Kamimura, H. (2007). Inhibitory potential of nonsteroidal anti-inflammatory drugs on UDP-glucuronosyltransferase 2B7 in human liver microsomes. Eur. J. Clin. Pharmacol. 63 211-216.

Miners, J. O., Knights, K. M., Houston, J. B., and Mackenzie, P. I. (2006). In vitro-in vivo correlation for drugs and other compounds eliminated by glucuronidation in humans: pitfalls and promises. Biochem. Pharmacol. 71, 1531-1539.
Nakamura, A., Nakajima, M., Yamanaka, H., Fujiwara, R., and Yokoi, T. (2008). Expression of UGT1A and UGT2B mRNA in human normal tissues and various cell lines. Drug Metab. Dispos. 36, 1461-1464.

Park, J., Park, S., Lho, D., Choo, H. P., Chung, B., Yoon, C., et al. (1990). Drug testing at the 10th Asian games and 24th Seoul Olympic games. J. Anal. Toxicol. 14, 66-72.

Schulze, J. J., Lorentzon, M., Ohlsson, C., Lundmark, J., Roh, H. K., Rane, A., et al. (2008a). Genetic aspects of epitestosterone formation and androgen disposition: influence of polymorphisms in CYP17 and UGT2B enzymes. Pharmacogenet. Genomics 18, 477-485.

Schulze, J. J., Lundmark, J., Garle, M., Skilving, I., Ekstrom, L., and Rane, A. (2008b). Doping test results dependent on genotype of uridine diphospho-glucuronosyl transferase 2B17, the major enzyme for testosterone glucuronidation. J. Clin. Endocrinol. Metab. 93, 2500-2506.

Schulze, J. J., Thorngren, J. O., Garle, M., Ekstrom, L., and Rane, A. (2011). Androgen Sulfation in Healthy UDP-glucuronosyl transferase 2B17 enzyme-deficient men. J. Clin. Endocrinol. Metab. 96, 3440-3447.

Sten, T., Bichlmaier, I., Kuuranne, T., Leinonen, A., Yli-Kauhaluoma, J., and Finel, M. (2009a). UDPglucuronosyltransferases (UGTs) 
2B7 and UGT2B17 display converse specificity in testosterone and epitestosterone glucuronidation, whereas UGT2A1 conjugates both androgens similarly. Drug Metab. Dispos. 37, 417-423.

Sten, T., Finel, M., Ask, B., Rane, A., and Ekstrom, L. (2009b). Non-steroidal anti-inflammatory drugs interact with testosterone glucuronidation. Steroids 74, 971-977.

Turgeon, D., Carrier, J. S., Levesque, E., Hum, D. W., and Belanger, A. (2001). Relative enzymatic activity, protein stability, and tissue distribution of human steroid-metabolizing UGT2B subfamily members. Endocrinology 142, 778-787.
Van der Logt, E. M., Roelofs, H. M., Van Lieshout, E. M., Nagengast, F. M., and Peters, W. H. (2004). Effects of dietary anticarcinogens and nonsteroidal anti-inflammatory drugs on rat gastrointestinal UDP-glucuronosyltransferases. Anticancer Res. 24, 843-849.

WADA. (2011). Laboratory Testing Figures. Montreal: Reported by Accredited Laboratories.

Warden, S. J. (2009). Prophylactic misuse and recommended use of nonsteroidal anti-inflammatory drugs by athletes. Br. J. Sports Med. 43, 548-549.

Zhou, S. F., Zhou, Z. W., and Huang, M. (2010). Polymorphisms of human cytochrome P450 2C9 and the functional relevance. Toxicology 278 165-188.

Conflict of Interest Statement: The authors declare that the research was conducted in the absence of any commercial or financial relationships that could be construed as a potential conflict of interest.

Received: 09 January 2013; accepted: 18 April 2013; published online: 16 May 2013.

Citation: Lundmark J, Gårevik N, Thörngren J-O, Garle M, Ekström L, Rane A and Schulze JJ (2013)
Non-steroidal anti-inflammatory drugs do not influence the urinary testosterone/epitestosterone glucuronide ratio. Front. Endocrinol. 4:51. doi 10.3389/fendo.2013.00051

This article was submitted to Frontiers in Experimental Endocrinology, a specialty of Frontiers in Endocrinology.

Copyright (c) 2013 Lundmark, Gårevik, Thörngren, Garle, Ekström, Rane and Schulze. This is an open-access article distributed under the terms of the Creative Commons Attribution License, which permits use, distribution and reproduction in other forums, provided the original authors and source are credited and subject to any copyright notices concerning any third-party graphics etc. 